\title{
Experiencia de Mobile Learning, evaluación formativa y compartida en el Grado en Magisterio en Educación Primaria en la Universidad de Cantabria
}

\section{Mobile Learning, formative and shared assessment experience in the Degree in Teaching in Primary Education in the University of Cantabria}

\author{
Mikel Pérez-Gutiérrez ${ }^{1}$, Carlos Cobo-Corrales ${ }^{2}$
}

\begin{abstract}
Fecha de recepción: 24/01/2019; Fecha de revisión: 21/02/2019; Fecha de aceptación: 25703/2019 Cómo citar este artículo:

Pérez-Gutiérrez, M., \& Cobo-Corrales, C. (2019). Experiencia de Mobile Learning, evaluación formativa y compartida en el Grado de Magisterio en Educación primaria en la Universidad de Cantabria. Revista de Innovación y Buenas Prácticas Docentes, 8, 15-26.
\end{abstract}

Autor de correspondencia: Mikel.perez@unican.es

\section{Resumen:}

Se describe una experiencia de Mobile learning, evaluación formativa y compartida en una asignatura del Grado en Magisterio en Educación Primaria de la Universidad de Cantabria, con el objeto de actualizar la metodología docente, fomentar las competencias digitales demandas por la sociedad y mejorar los procesos de enseñanza-aprendizaje. La experiencia se dividió en dos fases, la primera dedicada a la preparación de las aplicaciones informáticas y materiales; y la segunda a la puesta en práctica y evaluación de la experiencia. Los beneficios mejor valorados fueron la utilidad, las ayudas recibidas por el profesor y la satisfacción global con la experiencia. Los principales inconvenientes fue la asistencia obligatoria, activa y de forma continuada a las clases. En conclusión, el alumnado presentó una visión favorable del sistema de evaluación aplicado y la asignatura.

Palabras clave: Innovación Pedagógica, Tecnologías de la Información y de la Comunicación, Enseñanza Superior, Evaluación Formativa

\begin{abstract}
:
It describes a Mobile Learning, formative and shared assessment experience carried out in a course within the Degree in Teaching in Primary Education in the University of Cantabria, with the aim of updating the teaching methodology used, promoting the digital competences demanded by the current society and enhancing the teaching-learning processes. Thus, this experience was divided in two phases, first one related to the preparation of the software and teaching materials; and second one to the application and evaluation of the experience. The best-valued benefits were the utility, aids received from the teacher and the general satisfaction with the experience. The main disadvantages were the compulsory, active and continued assistance to lessons. In summary, students showed a positive perception of the assessment system applied and the course.
\end{abstract}

Key Words: Educational Innovation, Information and Communication Technologies, Higher Education, Formative Evaluation

\footnotetext{
1 Universidad de Cantabria (España), mikel.perez@unican.es; https://orcid.org/0000-0001-9169-958X. 2 Universidad de Cantabria (España), carlos.cobo@unican.es; https://orcid.org/0000-0002-2155-1309.
} 


\section{INTRODUCCIÓN}

En la actual sociedad en la que vivimos, cada vez es más importante la presencia y el papel que desempeñan las tecnologías de la información y la comunicación en nuestras vidas, por ejemplo, con la aparición de los smartphones, la emergencia de la inteligencia artificial y el internet de las cosas, la economía 2.0 o eCommerce, la eAdministración, la aparición de los vehículos autónomos o el crecimiento de los eSports, pudiendo definirse como una sociedad digital (Fundación Telefónica, 2018). Esta hiperrealidad, con su aumento de significadores, medios e información, "disminuye nuestra capacidad para encontrar significado o generar pasión por el compromiso" (Steinberg \& Kincheloe, 2000, p. 22). Tales cambios sociales y tecnológicos requieren una alfabetización en esos nuevos medios, no como una adición al currículum tradicional, "sino en una destreza básica necesaria para negociar la propia identidad, los valores y el bienestar en la hiperrealidad empapada de poder" (Steinberg \& Kincheloe, 2000, p. 23). Debido a la importancia que esta sociedad asigna a la información y al conocimiento, implicando la multiplicación del poder de la cualificación y la división social en torno a dicha cualificación, la educación adquiere una relevancia cada vez más importante (Fernández Enguita, 2006).

Las características de dicha sociedad quedan reflejadas en los siguientes datos. A nivel mundial, el 66\% de la población cuenta con un teléfono móvil (Ditrendia, 2017) y el número de líneas móviles supera al número de habitantes en el planeta en el año 2017 con un total de 7740 millones de suscripciones (Fundación Telefónica, 2018). De forma similar, a nivel nacional existen más de 18 millones de hogares con fibra óptica y el $86 \%$ de los jóvenes entre 16 y 29 años posee un smartphone, utilizando este dispositivo a diario para acceder a internet (Fundación Telefónica, 2018).

Pese al pesimismo educativo actual, los continuos cambios sociales provocan que la institución escolar tenga una importancia económica y social nunca antes vista (Fernández Enguita, 2006). Como docentes debemos trabajar los nuevos medios en la escuela para convertirlos en herramientas para la mejora y el cambio social. No basta con enseñar nuevos códigos o lenguajes, es necesario enseñar el uso de estos avances con análisis crítico y reflexión, no confundir los medios con el fin. Estos cambios en la epistemología y la cultura provocan la transformación en el modo de concebirlo, lo que a su vez conlleva transformaciones sociales y económicas que fuerzan el cambio de las escuelas (Rodríguez Romero, 2003). La escuela debería ser un actor social y no un mero resultado del contexto imperante, conceptualizado por algunos autores como la Tercera Revolución Industrial (Fernández Enguita, 2006).

Esta situación social también se ve reflejada en las aulas de Educación Superior, donde ordenadores portátiles, smartphones, pizarras digitales y otros dispositivos electrónicos forman parte de las dinámicas de clase (Marciales Vivas, 2012). A tenor de los datos mostrados, el smartphone es una de las principales herramientas que podemos aplicar en nuestro contexto educativo debido fundamentalmente a su penetración social. De esta manera, el Mobile Learning puede definirse como la "modalidad educativa que facilita la construcción del conocimiento, la resolución de problemas de aprendizaje y el desarrollo de destrezas o habilidades diversas de forma autónoma y ubicua gracias a la mediación de dispositivos móviles portables" (Brazuelo Grund \& Gallego Gil, 2011, p. 17). Junto con las ventajas funcionales del Mobile Learning, principalmente su accesibilidad y penetración social (Fundación Telefónica, 2018; ISEA, 2009), las principales razones para seleccionar el Mobile Learning como experiencia educativa innovadora fueron su penetración a nivel social, especialmente en los jóvenes entre 16 y 29 años con un $86 \%$ de ellos utilizando un smartphone (Fundación Telefónica, 2018), su mejora del aprendizaje centrado en el alumno (Corbeil \& Valdes-Corbeil, 2007), su carácter interactivo (González-Fernández \& SalcinesTalledo, 2015), su mejora de la participación, motivación y comunicación entre profesor y alumno (Corbeil \& Valdes-Corbeil, 2007; ISEA, 2009) además de la satisfacción general del alumnado (infoNet, 2012). Además, estas ventajas han sido demostradas 
dentro del contexto educativo en diversas investigaciones (Corbeil \& Valdes-Corbeil, 2007; Marcos López, Támez Almaguer, \& Lozano Rodríguez, 2009; Monguillot Hernando, González Arévalo, \& Guitert i Catasús, 2015; Monguillot Hernando, González, \& Guitert i Catasús, 2017; Palazón Herrera, 2015; Ramos, Herrera, \& Ramírez, 2010).

Esta situación social y educativa es la que origina la presente experiencia, para aprovechar los avances tecnológicos, la predisposición y actitud favorable que tiene el alumnado para interaccionar con sus smartphones así como permitir aplicar una evaluación formativa y compartida. En este sentido, dicha evaluación se define como el proceso que tiene por objetivo mejorar los procesos de enseñanza-aprendizaje, apartándose del mero fin calificador y sirviendo al alumno para mejorar su proceso de aprendizaje, al profesor para mejorar su competencia docente y mejorar los procesos de enseñanza-aprendizaje desarrollados (López Pastor, 2017; Pérez Pueyo, Julián Clemente, \& López Pastor, 2009). En el ámbito de la Educación Física y a nivel nacional, la evaluación formativa y compartida ha sido principalmente tratada, aplicada e investigada por la Red de Evaluación Formativa y Compartida en Educación (REFYCE, 2019), estando algunos de sus últimos trabajos publicados dedicados a la percepción de competencia y motivación en los maestros de esta especialidad (Estevan, MolinaGarcía, García-Massó, \& Martos, 2018) o la adquisición de competencias docentes (Cañadas Martín, 2018). A nivel internacional, los estudios más recientes se han centrado en diferenciar la terminología y propósitos de la evaluación formativa respecto a la evaluación para el aprendizaje (Chng \& Lund, 2018) o estudiar las diferentes versiones que existen de la evaluación formativa y sus resultados (Tolgfors, 2018). Aunque el Mobile Learning y los smartphones han sido utilizados en procesos de evaluación formativa y compartida tanto a nivel nacional (González-Fernández \& Salcines-Talledo, 2017; Ramírez García, 2015) como internacional (Hwang \& Chang, 2011; Nikou \& Economides, 2018), en el ámbito de la Educación Física no se encontraron experiencias similares.

Por lo tanto, esta investigación pretendió actualizar la metodología docente empleada, fomentar las competencias digitales demandas por la sociedad actual y mejorar los procesos de enseñanza-aprendizaje a través de la evaluación formativa y compartida. Así, el objetivo de este artículo es describir una experiencia de Mobile Learning aplicada en una asignatura perteneciente al Grado en Magisterio en Educación Primaria de la Universidad de Cantabria durante el curso académico 2017-2018 y conocer la percepción del alumnado respecto a dicha experiencia y el sistema de evaluación formativa y compartida desarrollado en dicha asignatura.

\section{DESARROLLO DE LA EXPERIENCIA DE INNOVACIÓN}

La elección del Mobile Learning como experiencia de innovación pedagógica fue debida a sus posibilidades para mejorar la participación del alumnado en el desarrollo de las clases, aumentar la motivación hacia los contenidos de la asignatura además de permitir la evaluación formativa y compartida del alumnado para que éste sea consciente y autónomo de su propio proceso de enseñanza-aprendizaje. Por su parte, la elección de la evaluación formativa y compartida descansó en los múltiples beneficios señalados que se pueden resumir en la mejora de la motivación del alumnado, que aprende más y mejor, facilita el desarrollo de su capacidad de análisis crítico reflexivo y autocrítica además de su autonomía y responsabilidad (López Pastor, 2009). Para el docente, le permite la corrección a tiempo de problemas, con lo cual mejora el proceso de 
enseñanza-aprendizaje, y se constituye como una experiencia de aprendizaje en sí misma. Además, fruto de todos estos beneficios, mejora el rendimiento académico en las asignaturas donde se implanta (López Pastor, 2009). Por lo tanto, el Mobile Learning así como la evaluación formativa y compartida son experiencias y herramientas con gran potencial, tanto para alumnos y profesores como para la propia institución educativa. No se busca sin más poner en marcha ideas de terceros, sino que es un proceso donde los propios profesores debemos formarnos, aprendizaje que se quiere extrapolar a los futuros docentes, ya que no puede existir un desarrollo curricular sin existir un desarrollo del propio docente. Las ideas, sentido de responsabilidad y compromiso de los docentes con la "oferta eficaz de experiencias educativas a sus alumnos se refuerza de forma significativa cuando son dueños de las ideas que plasman y autores de los medios que traducen esas ideas a la práctica de clase" (MacDonald, 1999, p. 12). Debemos evitar que los nuevos medios tecnológicos se conviertan en una versión digital de los libros de texto, instrumento que colonizó el trabajo docente y se convirtió en el auténtico currículum escolar (Martínez Bonafé \& Adell, 2004).

La presente experiencia se desarrolló en la asignatura de Didáctica de la Educación Física en Educación Primaria, asignatura obligatoria de primer curso del grado en Magisterio en Educación Primaria de la Universidad de Cantabria, durante el segundo cuatrimestre del curso académico 2017-2018. El Mobile Learning fue aplicado en dos de los cuatro grupos que existen en la asignatura, incluyendo un total de 108 alumnos. Cabe resaltar que el desarrollo de la asignatura incluyó otras actividades y estrategias de enseñanza-aprendizaje para el desarrollo de los contenidos teóricoprácticos que componen la asignatura, tal como indica la propia guía docente de la misma (UC, 2019).

La experiencia se desarrolló a través de la utilización de tres aplicaciones informáticas (Edpuzzle, Kahoot y Mentimeter). Mientras que la aplicación Mentimeter permite crear y aplicar cuestionarios de conocimientos previos, Kahoot ofrece la posibilidad de realizar pruebas de selección múltiple, ambas aplicaciones orientadas a la evaluación formativa y compartida. Por su parte, la aplicación Edpuzzle permite la edición y preparación de vídeos educativos con el objetivo de que el alumnado sea consciente y autónomo de su propio proceso de enseñanza-aprendizaje. Por lo tanto, al comienzo de cada nuevo tema de la asignatura se desarrolló de manera presencial una evaluación diagnóstica de los conocimientos previos del alumnado a través del Mentimeter. Esta evaluación inicial también fue utilizada para comenzar a relacionar sus conocimientos previos con los contenidos teóricos. En la clase posterior y tras haber finalizado cada tema, se realizó una evaluación formativa y compartida a través de la aplicación Kahoot con la realización de preguntas tipo test de selección múltiple. Finalmente, como tareas no presenciales, se editaron 7 videos en la aplicación Edpuzzle relacionados con los temas de la asignatura, para desarrollar su capacidad crítica y de reflexión, proporcionando una evaluación formativa de los mismos.

El proceso de innovación aplicado puede dividirse en dos fases, la primera de ellas dedicada a la preparación de las aplicaciones y materiales (previa al comienzo de las clases) y la segunda dedicada a la puesta en práctica y evaluación de la experiencia (durante el cuatrimestre). De forma detallada, en la primera fase se procedió al registro y creación de cuentas en las aplicaciones informáticas Edpuzzle, Kahoot y Mentimeter. Solo en Mentimeter se contrató la versión educativa (Mentimeter Education Pro) para disponer de todas sus funcionalidades. Posteriormente se crearon los cuestionarios de conocimientos previos de cada uno de los nueve temas de la asignatura en Mentimeter, las pruebas de selección múltiple para evaluar los contenidos de dichos temas en Kahoot así como los videos de análisis y evaluación de los temas seleccionados (7) en Edpuzzle. Respecto a la temporalización de estas actividades, los cuestionarios de conocimientos previos (Mentimeter) se aplicaron al comienzo de cada tema, mientras que las pruebas de selección múltiple (Kahoot) se desarrollaron la clase siguiente a la finalización de cada tema. Finalmente, los videos de análisis y evaluación (Edpuzzle) permanecieron abiertos desde el comienzo del curso y se fueron cerrando 
progresivamente a lo largo de todo el cuatrimestre para que el alumnado pudiera dosificar su propio proceso de enseñanza-aprendizaje. La Figura 1 ofrece un listado de las actividades desarrolladas junto con su temporalización.

\begin{tabular}{ll}
\hline Fecha & Actividad \\
\hline 08-02-2018 & Mentimeter Tema 1 \\
13-02-2018 & Kahoot Tema 1. Mentimeter Tema 2 \\
19-02-2018 & Edpuzzle Tema 1 \\
20-02-2018 & Kahoot Tema 2. Mentimeter Tema 3 \\
26-02-2018 & Edpuzzle Tema 2 \\
27-02-2018 & Mentimeter Tema 4 \\
05-03-2018 & Edpuzzle Tema 3 \\
06-03-2018 & Mentimeter Tema 5 \\
13-03-2018 & Kahoot Tema 5. Mentimeter Tema 6 \\
20-03-2018 & Kahoot Tema 6. Mentimeter Tema 7 \\
26-03-2018 & Edpuzzle Tema 4 \\
27-03-2018 & Kahoot Tema 7. Mentimeter Tema 8 \\
16-04-2018 & Edpuzzle Tema 8 \\
23-04-2018 & Edpuzzle Tema 9 \\
24-04-2018 & Kahoot Tema 8. Mentimeter Tema 9 \\
07-05-2018 & Edpuzzle Tema 5 \\
15-05-2018 & Kahoot Tema 4. Kahoot Tema 9 \\
\hline
\end{tabular}

Figura 1. Cronograma de actividades de Mobile Learning desarrolladas.

Durante la segunda fase se aplicaron las tareas desarrolladas en las aplicaciones informáticas Kahoot, Mentimeter y Edpuzzle. Además, tanto al inicio como al final del cuatrimestre se evaluó la experiencia de innovación dedicada al Mobile Learning así como su evaluación mediante la aplicación del Cuestionario sobre la experiencia de "buena práctica" y la asignatura, creado por la Red de Evaluación Formativa y Compartida en Educación (REFYCE) y basado en el cuestionario sobre metodología y evaluación en formación inicial en Educación Física validado por Castejón Oliva, Santos Pastor, and Palacios Picos (2015). El cuestionario está dividido en tres partes, la primera de ellas dedicada a presentar el objetivo del cuestionario y recabar los datos identificativos del encuestado. La segunda parte incluye 13 ítems sobre la experiencia innovadora, mientras que la tercera parte está compuesta por 32 ítems sobre la asignatura de forma global. Los ítems de esta tercera parte pueden agruparse en las ventajas que reconoce el alumnado respecto al sistema de evaluación aplicado (ítems 1 a 18) y los inconvenientes (ítems 19 a 32). Cada uno de los ítems se valora en una escala Likert de 5 puntos, siendo 1 nada y 5 mucho.

\section{RESULTADOS}

Los alumnos que participaron de la experiencia innovadora fueron un total de 105, mientras que los otros tres alumnos optaron por el examen final de la asignatura. Además, un $69.6 \%$ de los alumnos declararon no haber realizado una experiencia similar con anterioridad, un dato llamativo teniendo en cuenta la presencia social de los dispositivos móviles.

Respecto a la evaluación del Mobile Learning como experiencia innovadora, la Tabla 1 resume los valores medios obtenidos en cada ítem.

Tabla 1. 
Media y desviación típica (DT) de los ítems que componen el cuestionario sobre la experiencia de "buena práctica" y su evaluación.

\begin{tabular}{lcc}
\hline \multicolumn{1}{c}{ Items } & Media & DT \\
\hline $\begin{array}{l}\text { ¿Se ha negociado la utilización de esta experiencia en la asignatura al } \\
\begin{array}{l}\text { comienzo del curso } \\
\text { ¿Crees que esta experiencia te ha ayudado a adquirir competencias }\end{array}\end{array}$ & 2.9 & 1.3 \\
profesionales? & 3.9 & 0.7
\end{tabular}

Tabla 2.

Media y desviación típica (DT) de los ítems que componen el cuestionario sobre la experiencia de "buena práctica" y su evaluación (continuación).

\section{Items}

¿La evaluación que se ha planteado favorece la adquisición de las competencias profesionales

¿Qué es lo más útil que has aprendido? Que es una experiencia innovadora.

Media DT

$3.9 \quad 0.7$

¿Qué es lo más útil que has aprendido? Que es una experiencia efectiva.

¿Qué es lo más útil que has aprendido? Que es una experiencia sostenible.

¿Qué es lo más útil que has aprendido? Que es una experiencia replicable.

¿Cómo valoras las ayudas recibidas por el docente?

¿Cómo valoras las ayudas recibidas por los compañeros?

Señala la satisfacción global en relación con la experiencia

Señala la satisfacción global en relación con la evaluación de la experiencia

¿Cuál es el grado de dificultad de la experiencia?

Fuente: Elaboración propia.

Entre los elementos más valorados que destacan los alumnos sobre el Mobile Learning aparecen la utilidad, las ayudas recibidas por el profesor y la satisfacción global con la experiencia, indicando bastante acuerdo con dichas afirmaciones (4.1 respectivamente). Por el contrario, el alumnado señaló que el grado de dificultad de la experiencia fue entre poco y algo (2.6). Mientras que la negociación de la utilización de la experiencia al comienzo del curso fue media (2.9). En el resto de ítems, el alumnado estuvo bastante de acuerdo con las afirmaciones presentadas, destacando el aporte tanto de la experiencia como de la evaluación utilizada para su formación docente (3.9 respectivamente), concretamente en la adquisición de competencias profesionales.

Respecto a la evaluación de la asignatura de forma global, la Tabla 2 muestra la opinión del alumnado respecto a las ventajas que reconocen en relación al sistema de evaluación aplicado. El alumnado estuvo entre bastante y muy de acuerdo con el trabajo en equipo de forma colaborativa (4.5) así como con el aprendizaje activo y la posibilidad de corregir errores (4.3 respectivamente) en el desarrollo de la asignatura. Además, el alumnado estuvo bastante de acuerdo con el resto de ítems. Sin embargo, solo estuvo algo de acuerdo con que la asignatura ofreciera un seguimiento más individualizado (3.2). Estos resultados indican una visión general del alumnado favorable al sistema de evaluación aplicado, señalando más aprendizajes funcionales y significativos, una asignatura más motivante, centrada en el proceso y en el trabajo diario, lo que supuso un aumento de la calidad de los trabajos exigidos, pero también conllevando una mayor responsabilidad.

Ontoria Peña, Molina Rubio, and Luque Sánchez (1997) exploraron los conflictos (cognitivos y de autoconcepto) que viven los estudiantes de Magisterio al pasar de metodologías que enfatizan la labor directiva del profesor a propuestas centradas en un trabajo más autónomo del alumno. Concluyeron que los estudiantes tienen una experiencia formativa marcada por un planteamiento tradicional y eso les origina sentimientos de inseguridad y desconcierto ante innovaciones metodológicas. Comentar 
aquí que nuestra experiencia de Mobile Learning es solo un aspecto más de nuestra materia, coexistiendo con otras estrategias y tareas de enseñanza-aprendizaje como clases magistrales, talleres, trabajos grupales, prácticas dirigidas o debates, entre otros. Nuestra intención era darle su justa importancia, que no se viesen estas actividades como meras acciones a completar ni tampoco como sobrecarga para la materia.

También hemos tratado de evitar asociar el carácter alternativo y novedoso de la propuesta de Mobile Learning como una herramienta puntual y anecdótica insertada en un sistema de evaluación donde el examen sigue contando con un rol importante, "un estereotipo que suele ser habitual y que está relacionado con el hecho de que se otorga [por parte del alumnado] una menor importancia a los trabajos de una asignatura cuando existe un examen final puntuado con más nota" (Herrada Valverde, Cobo Corrales, \& Herrada Valverde, 2008, p. 179).

Tabla 3.

Media y desviación típica (DT) de los ítems que componen las ventajas relativas al sistema de evaluación aplicado del cuestionario sobre la asignatura de forma global.

\begin{tabular}{lcc}
\multicolumn{1}{c}{ Items } & Media & DT \\
\hline 01. Ofrece alternativas a todos los estudiantes & 4.1 & 0.7 \\
02. Hay un contrato previo, negociado y consensuado del sistema de & 4 & 1.1 \\
evaluación & 3.9 & 0.6 \\
03. Está centrado en el proceso, importancia del trabajo diario & 4.3 & 0.6 \\
04. El estudiante realiza un aprendizaje activo & 4.5 & 0.7 \\
05. Se plantea el trabajo en equipo de forma colaborativa & 4 & 0.8 \\
06. El alumno/a está más motivado, el proceso de aprendizaje es más & 3.8 & 0.9 \\
motivador & 3.6 & 0.9 \\
07. La calificación es más justa & 3.8 & 0.6 \\
08. Mejora la tutela académica (seguimiento y ayuda al alumno/a) & 3.8 & 0.6 \\
09. Permite aprendizajes funcionales & 3.8 & 0.7 \\
10. Genera aprendizajes significativos & 3.7 & 0.8 \\
11. Se aprende mucho más & 4 & 0.8 \\
12. Mejora la calidad de los trabajos exigidos & 4.1 & 0.7 \\
13. Hay interrelación entre teoría y práctica & 4.1 & 0.7 \\
14. Evalúa todos los aspectos posibles (en referencia al saber, saber hacer y y & 4.3 & 0.9 \\
saber estar y ser)) & 3.2 & 0.9 \\
15. Hay retroalimentación en documentos y actividades & 3.9 & 0.8 \\
16. Hay posibilidad de corregir errores en documentos y actividades & & \\
17. Se da un seguimiento más individualizado & &
\end{tabular}

Fuente: Elaboración propia.

En relación a los inconvenientes del sistema de evaluación aplicado, la Tabla 3 muestra la percepción del alumnado. Estos estuvieron bastante de acuerdo con que dicho sistema requirió una asistencia obligatoria, activa y de forma continuada (4 respectivamente), exigiendo por lo tanto un mayor esfuerzo (3.7) y comprensión previa (3.4). Sin embargo, el planteamiento de los contenidos y actividades de la asignatura parece adecuado ya que el alumnado mostró poco acuerdo con que existiera una desproporción entre los trabajos propuestos y los créditos de la asignatura (2.1). Además de no considerar injusto el sistema de evaluación utilizado frente a otro tipo de procesos de evaluación (1.7). Esta actitud favorable del alumnado hacia el sistema de evaluación también quedó corroborada al considerar que las correcciones fueron claras 
(2.1), apenas percibieron que la valoración del trabajo fuera subjetiva (2.2) o que se generase inseguridad o incertidumbre en este sistema (2.5).

Tabla 4.

Media y desviación típica (DT) de los ítems que componen los inconvenientes relativos al sistema de evaluación aplicado del cuestionario sobre la asignatura de forma global.

\begin{tabular}{lcc}
\hline \multicolumn{1}{c}{ Items } & Media & DT \\
\hline 19. Exige una asistencia obligatoria y activa & 4 & 0.9 \\
20. Tiene una dinámica de trabajo poco conocida, falta de hábito & 2.7 & 1 \\
21. Exige continuidad & 4 & 0.8 \\
22. Hay que comprenderlo previamente & 3.4 & 1 \\
23. Exige un mayor esfuerzo & 3.7 & 0.9 \\
24. Existe dificultad para trabajar en grupo & 2.6 & 1.2 \\
25. Se puede acumular mucho trabajo al final & 3.2 & 1.2 \\
26. Existe una desproporción trabajo/créditos & 2.1 & 1 \\
Tabla 5. & & \\
Media y desviación típica (DT) de los ítems que componen los inconvenientes relativos al sistema \\
de evaluación aplicado del cuestionario sobre la asignatura de forma global (continuación). \\
$\quad$ Items & Media & DT \\
& 2.3 & 1 \\
27. El proceso de calificación es más complejo y, a veces, poco claro & 1.7 & 0.9 \\
29. Es injusto frente a otros procesos de evaluación & 2.1 & 1.1 \\
30. Las correcciones han sido poco claras & 2.2 & 1 \\
31. La valoración del trabajo es subjetiva & 3 & 1 \\
32. Exige participar en mi propia evaluación (autoevaluarse) &
\end{tabular}

Los resultados mostrados en la presente experiencia de Mobile Learning y la evaluación aplicada durante el desarrollo de la asignatura muestran bastante satisfacción del alumnado con ambos aspectos a nivel general. Como inconveniente más puntuado está la exigencia de asistir obligatoriamente, aunque este aspecto resulta necesario para desarrollar la evaluación continua que se aplica como norma general en todas las asignaturas y que queda recogida en la normativa de los sistemas de evaluación de la Universidad de Cantabria (UC, 2018). Además, los alumnos señalan un claro beneficio para su formación y competencias docentes, aunque dicho beneficio requiere un trabajo y esfuerzo constante a lo largo de todo el proceso, así como al comienzo de la asignatura para entender el planteamiento pedagógico realizado.

\section{CONCLUSIONES}

La preparación y puesta en práctica de una experiencia de Mobile Learning supone una planificación y organización detallada de los contenidos de la asignatura para adecuar las actividades de Mobile Learning al desarrollo de los mismos, un trabajo previo considerable al comienzo de la asignatura para la creación de cuentas, selección y edición de videos y materiales relacionados con los contenidos de la asignatura, así como cierta experiencia en el manejo de las aplicaciones informáticas utilizadas (Edpuzzle, Kahoot y Mentimeter). Estos condicionantes implican un amplio conocimiento y dominio de los contenidos de la asignatura por parte del profesorado para seleccionar las actividades más adecuadas a desarrollar con cada aplicación informática, así como agilizar la preparación y evaluación de dichas actividades.

La cantidad de alumnos que no habían vivenciado una experiencia de Mobile Learning, el alto grado de satisfacción tanto con la experiencia como con el sistema de evaluación utilizado y su contribución a la adquisición de competencias docentes, la dificultad media percibida por los alumnos para esta experiencia, así como la promoción del trabajo en equipo, el aprendizaje activo y la evaluación formativa y compartida durante todo el proceso son algunas de las causas más importantes que justifican la 
preparación y desarrollo de esta innovación pedagógica centrada en el Mobile Learning. Aunque a nivel general esta experiencia conlleva una carga elevada de trabajo para el profesorado, sobre todo antes del comienzo de la asignatura, le permite durante el desarrollo del cuatrimestre centrarse en la revisión y corrección de tareas, esto es, en ofrecer una evaluación formativa y compartida al alumnado que le permita avanzar consciente de su ritmo de aprendizaje.

Respecto a las aplicaciones utilizadas, la inclusión del Mentimeter facilitó la participación de todo el alumnado en la evaluación diagnóstica relativa a los contenidos de la asignatura, sirviendo sus conocimientos previos como puerta de acceso a los contenidos teóricos de la asignatura. La utilización del Kahoot conllevó un aumento de la participación y motivación del alumnado hacia los contenidos de la asignatura, mostrando una actitud activa que se reflejó en la demanda de un Kahoot en cada clase. Finalmente, el uso de Edpuzzle permitió la entrega de una retroalimentación inmediata tras la realización de las actividades, convirtiéndose en una evaluación formativa que dirigió el proceso de enseñanza-aprendizaje del alumnado.

A nivel personal, la experiencia ha sido muy enriquecedora para ambos profesores ya que nos ha permitido mejorar nuestra coordinación, enriquecer nuestra formación, mejorar nuestra capacidad de análisis y reflexión sobre los contenidos de la asignatura, discutir nuestra propia actuación docente y nuestro método de evaluación, así como dinamizar nuestras clases.

Sin embargo, esta experiencia presentó algunas limitaciones que merece la pena destacar. En primer lugar, se detectaron algunos problemas relacionados con la aplicación Edpuzzle, concretamente el olvido de las credenciales por parte de los alumnos para acceder a la aplicación, requiriendo la recuperación de la contraseña o incluso la creación de nuevas cuentas para completar las actividades. En segundo lugar, aparecieron problemas de visionado de los videos de Edpuzzle a través del smartphone y/o la tablet, que parecen responder a problemas técnicos de la propia aplicación. En tercer lugar, la organización y corrección de tareas fue dificultosa ya que los alumnos de ambos grupos fueron asignados a una misma clase de Edpuzzle, por lo que requirió la edición de los nombres de usuario de los alumnos para identificarlos y organizarlos adecuadamente. $Y$, en cuarto lugar, la utilización de videos disponibles en YouTube $u$ otras plataformas posibilitó que los alumnos, en algunos casos, vieran los videos editados en Edpuzzle en dichas plataformas antes de responder a las preguntas planteadas. Para solventar estos problemas, en el próximo curso académico se pretende recalcar la importancia de conservar las credenciales utilizadas en Edpuzzle para completar las actividades, promover la realización de las tareas en el ordenador para minimizar los errores técnicos de la aplicación, crear dos clases en Edpuzzle para que cada alumno se integre en su clase correspondiente, así como utilizar videos no disponibles en otras plataformas para que no existe una ventaja previa antes de responder a las preguntas planteadas en Edpuzzle.

Como ya hemos comentado anteriormente, el Mobile Learning era una parte más de la materia, no se centraba solamente en ella. Con esta innovación a medio plazo se pretende mejorar la totalidad de la asignatura y para ello se debe actuar en diferentes ámbitos y direcciones, ya que "las organizaciones cambian y se mejoran a través de procesos complejos en los que se encuentran mezclados los conocimientos, los afectos, las rutinas, las relaciones, los valores, las condiciones materiales, las influencias sociales..." (Santos Guerra, 2009, p. 171).

Además, esta experiencia de Mobile Learning puede enmarcarse dentro del paradigma de la escuela como eje de cambio, según el cual "el éxito y calidad de la educación y del cambio dependen de cómo se organiza y funcionan las escuelas" 
(Herrada Valverde et al., 2008, p. 170). Empleando elementos del paradigma cultural (la autonomía de los centros para la reconstrucción del currículum) y del paradigma socio crítico (entiende el centro educativo como una totalidad relacional) desarrolla un modelo donde el cambio se inicia desde abajo, no dependiendo de grandes reformas legales ni administrativas (Herrada Valverde et al., 2008).

En este sentido, y para finalizar, expresar que todas estas innovaciones deben tener un marcado fin social. Como formadores debemos ser conscientes de que nuestro trabajo no es simplemente preparar mano de obra para el incierto mundo laboral, el mismo que actualmente valora "capacidades y habilidades transversales, como la informática o los idiomas" (Fernández Enguita, 2006, p. 22), o bien unas competencias básicas (lectura, cálculo, utilización básica de la informática e inglés), así como competencias que favorezcan la innovación (aptitudes tecnológicas, sociales y organizativas) en ambos casos conducentes a permitir la rápida adaptación del trabajador a los cambios de puesto laboral y de entorno (Díez, 2007, p. 349). Debemos evitar que el proceso educativo se convierta "en una labor meramente técnica. Se le cercena y se niega el eminente carácter social y político del acto educativo que implica conflictos de intereses, valores e ideales" (Díez, 2007, p. 359).

\section{REFERENCIAS}

Brazuelo Grund, F., \& Gallego Gil, Domingo J. (2011). Mobile learning: los dispositivos móviles como recurso educativo. Alcalá de Guadaíra (Sevilla): MAD.

Cañadas Martín, L. (2018). La evaluación formativa en la adquisición de competencias docentes en la formación inicial del profesorado de Educación Física. (Tesis Doctoral), Universidad Autónoma de Madrid, Madrid.

Castejón Oliva, F.J., Santos Pastor, M.L., \& Palacios Picos, A. (2015). Cuestionario sobre metodología y evaluación en formación inicial en educación física. Revista Internacional de Medicina y Ciencias de la Actividad Física y el Deporte, 15(58), 245-267. doi: http://dx.doi.org/10.15366/rimcafd2015.58.004

Corbeil, J. R., \& Valdes-Corbeil, M. E. (2007). Are You Ready for Mobile Leanrning? Educause Quarterly, (2), 51-58.

Chng, L. S., \& Lund, J. (2018). Assessment for Learning in Physical Education: The What, Why and How. Journal of Physical Education, Recreation and Dance, 89(8), 29-34. doi: 10.1080/07303084.2018.1503119

Díez, E. J. (2007). La globalización neoliberal y sus repercusiones en educación. Barcelona: El Roure.

Ditrendia (2017). Informe Mobile en España y en el Mundo 2017. Recuperado de https://www.amic.media/media/files/file_352_1289.pdf

Estevan, I., Molina-García, J., García-Massó, X., \& Martos, D. (2018). Efecto de la Intervención Docente en la Percepción de Competencia y Motivación de Futuros Maestros de Primaria en Educación Física Utilizando la Evaluación Formativa y Compartida. Estudios Pedagógicos, 44(2), 205-221.

Fernández Enguita, M. (2006). Educar en tiempos inciertos. Madrid: Morata.

Fundación Telefónica (2018). Sociedad Digital en España 2017. Barcelona: Ariel.

González-Fernández, N., \& Salcines-Talledo, I. (2015). El Smartphone en los procesos de enseñanza-aprendizaje-evaluación en Educación Superior. Percepciones de docentes y estudiantes. RELIEVE, Revista ELectrónica de Investigación y EValuación Educativa, 21(2), art. M3. doi: http://dx.doi.org./10.7203/relieve.21.2.7480

González-Fernández, N., \& Salcines-Talledo, I. (2017). Mobile learning en el Grado de Educación Infantil. Una buena práctica en la Universidad de Cantabria. Infancia, Educación y Aprendizaje (IEYA), 3(2), 71-76.

Herrada Valverde, G., Cobo Corrales, C., \& Herrada Valverde, R. I. (2008). Querer acabar antes de comenzar: las prisas por llegar al resultado. Enseñanza, 26, 165-185. 
Hwang, G. J., \& Chang, H. F. (2011). A formative assessment-based mobile learning approach to improving the learning attitudes and achievements of students. Computers \& Education, 56(4), 1023-1031. doi: 10.1016/j.compedu.2010.12.002

infoNet, JISC (2012). InfoKit de aprendizaje móvil. Recuperado de http://www.educoas.org/portal/la_educacion_digital/147/pdf/infokit.pdf

ISEA (2009). MOBILE LEARNING, Análisis prospectivo de las potencialidades asociadas al Mobile Learning. Recuperado de http://www.iseamcc.net/elSEA/Vigilancia_tecnologica/informe_4.pdf

López Pastor, V. M. (2009). Fundamentación teórica y revisión del estāo de la cuestión. En López-Pastor, V. M. (Ed.), Evaluación formativa y compartida en Educación Superior: Propuestas, técnicas, instrumentos y experiencias (pp. 45-64). Madrid: Narcea.

López Pastor, V. M. (2017). Evaluación formativa y compartida: evaluar para aprender y la implicación del alumnado en los procesos de evaluación y aprendizaje. En López Pastor, V. M. \& Pérez Pueyo, Á. (Eds.), Evaluación formativa y compartida en Educación: experiencias de éxito en todas las etapas educativas (pp. 34-68). León: Servicio de Publicaciones, Universidad de León.

MacDonald, B. (1999). Introducción crítica: de la innovación a la reforma. Un cambio de referencia para analizar el cambio. En Rudduck, J. (Ed.), Innovación y cambio: el desarrollo de la participación y la comprensión (pp. 9-25). Morón: Kikiriki Cooperación Educativa.

Marciales Vivas, G. P. (2012). Competencia informacional y brecha digital: preguntas y problemas emergentes derivados de investigación. Nómadas, 36, 127-143.

Marcos López, L., Támez Almaguer, R., \& Lozano Rodríguez, A. (2009). Aprendizaje móvil y desarrollo de habilidades en foros asincrónicos de comunicación. Comunicar: Revista Científica de Comunicación y Educación, XVII(33), 93-100.

Martínez Bonafé, J., \& Adell, J. (2004). Viejos y nuevos recursos y recnologías en el sistema educativo. En Gimeno Sacristán, J. \& Carbonell Seborroja, J. (Eds.), El sistema educativo: una mirada crítica. Madrid: CISSPRAXIS.

Monguillot Hernando, M., González Arévalo, C., \& Guitert i Catasús, M. (2015). Diseño de situaciones de aprendizaje mediadas por TIC en Educación Física. Revista Iberoamericana de Educación, 68(2), 63-81.

Monguillot Hernando, M., González, C., \& Guitert i Catasús, M. (2017). La colaboración virtual docente para diseñar situaciones de aprendizaje mediadas por TIC en Educación Física. Didacticae, 2, 6-23.

Nikou, S. A., \& Economides, A. A. (2018). Mobile-based assessment: A literature review of publications in major referred journals from 2009 to 2018. Computers and Education, 125, 101-119. doi: 10.1016/j.compedu.2018.06.006.

Ontoria Peña, A., Molina Rubio, A., \& Luque Sánchez, A. (1997). Autoconciencia del conflicto cognitivo ante la innovación metodológica en la formación inicial de maestros. Revista Electrónica Interuniversitaria de Formación del Profesorado, 1(0), 1-4.

Palazón Herrera, J. (2015). Aprendizaje móvil basado en microcontenidos como apoyo a la interpretación instrumental en el aula de música de secundaria. Píxel-Bit. Revista de Medios y Educación, 46, 119-136. doi: http://dx.doi.org/10.12795/pixelbit.2015.i46.08

Pérez Pueyo, Á., Julián Clemente, J. A., \& López Pastor, V M. (2009). Evaluación formativa y compartida en el Espacio Europeo de Educación Superior (EEES). En López-Pastor, V. M. (Ed.), Evaluación formativa y compartida en Educación 
Superior: Propuestas, técnicas, instrumentos y experiencias (pp. 19-43). Madrid: Narcea.

Ramírez García, A. (2015). Uso del smarthphone como instrumento de evaluación formativa Tendencias emergentes en evaluación formativa y compartida. En González Fernández, N., Salcines Talledo, I., \& García Ruiz, E. (Eds.), Tendencias emergentes en evaluación formativa y compartida en docencia. El papel de las nuevas tecnologías. Santander: Editorial de la Universidad de Cantabria.

Ramos, A. I., Herrera, J. A., \& Ramírez, Mª. S. (2010). Desarrollo de habilidades cognitivas con aprendizaje móvil: un estudio de casos. Comunicar: Revista Científica de Comunicación y Educación, XVII(34), 201-209.

REFYCE - Red de Evaluación Formativa Y Compartida en Educación (2019). ¿Quiénes somos? Recuperado de https://redevaluacionformativa.wordpress.com/

Rodríguez Romero, M ${ }^{\mathrm{a}}$. M. (2003). Las metamorfosis del cambio educativo. Tres Cantos: Akal.

Santos Guerra, M. A. (2009). La escuela que aprende. Retos, dificultades y esperanzas. En Santos Guerra, M. Á. (Ed.), Escuelas para la democracia. Cultura, organización y dirección de instituciones educativas (pp. 163-183). Madrid: Morata.

Steinberg, Sh. R., \& Kincheloe, J. L. (2000). Basta de secretos. Cultura infantil, saturación de información e infancia postmoderna. En S. R. Steinberg \& J.L. Kincheloe, J. L. (Eds.), Cultura infantil y multinacionales: la construcción de la identidad en la infancia (pp. 15-43). Madrid: Morata.

Tolgfors, B. (2018). Different versions of assessment for learning in the subject of physical education. Physical Education and Sport Pedagogy, 23(3), 311-327. doi: 10.1080/17408989.2018.1429589.

UC - Universidad de Cantabria (2018). Normas reguladoras de los procesos de evaluación en la Universidad de Cantabria. Recuperado de https://web.unican.es/estudios/Documents/SGA/Legislaci\%C3\%B3n\%20y\%20N ormativa/Normativa\%20Estudios\%20de\%20Grado/Normativa_Evaluaci\%C3\%B 3n_CG.pdf

UC - Universidad de Cantabria (2019). Guía docente de la asignatura. G308-Didáctica de la Educación Física en Educación Primaria. Recuperado de https://web.unican.es/estudios/Documents/Guias/2018/es/G308.pdf 